\title{
Linear Discrete-time Systems with Markovian Jumps and Mode Dependent Time-delay: Stability and Stabilizability
}

\author{
E. K. BOUKAS ${ }^{a, *}$, P. SHI ${ }^{b, \dagger}$, M. KARAN ${ }^{c, \ddagger}$ and C. Y. KAYA ${ }^{d, \S}$ \\ ${ }^{a}$ Mechanical Engineering Département, École Polytechnique de Montréal, P. O. Box 6079, Station, \\ "centre-ville", Montréal, Québec, H3C 3A7, Canada; 'Land Operations Division, Defence Science \\ and Technology Organisation, P. O. Box 1500, Salisbury, 5108 SA, Australia; ${ }^{\circ}$ CRC for Sensor Signal \\ and Information Processing (CSSIP), SPRI Building, Technology Park, Mawson Lakes, SA 5095 \\ Australia; ${ }^{d}$ School of Mathematics, University of South Australia, Mawson Lakes, SA 5095, Australia.
}

(Received 7 August 2001; In final form 21 January 2002)

This paper considers stochastic stability and stochastic stabilizability of linear discrete-time systems with Markovian jumps and mode-dependent time-delays. Linear matrix inequality (LMI) techniques are used to obtain sufficient conditions for the stochastic stability and stochastic stabilizability of this class of systems. A control design algorithm is also provided. A numerical example is given to demonstrate the effectiveness of the obtained theoretical results.

Key words: Discrete-time systems, time-delay, stochastic systems, stability, stabilizability

\section{INTRODUCTION}

Time-delay is an important factor that may affect the performance of dynamical systems. It can even, in some situation, causes instability of a system that we would like to control if the presence of such time-delay during the design phase is not taken into account. For deterministic linear systems with time-delay, we have seen an increasing interest during the last two decades. There are numerous results in the literature on time-delay systems, see for example, [11] and the references therein. However, most results are on deterministic continuous-time linear systems with time-delay. Stability, stabilizability and control problems for this class of systems have been studied and numerous results are available in the literature such as $[5,6,8-10,12,14,15]$. However, for deterministic class of discrete-time linear systems with time-delay only few results have been reported in the literature. We believe that the main reason for this is that these systems can be transformed to equivalent systems without time-delay and then current results on stability, stabilizability and control design can be applied. For more information on this class of systems, we refer reader to Boukas and Liu [3] and the references therein.

The system we are studying in this paper is referred to as the discrete-time Markovian jump linear systems with time-delay. It has two components in the state vector. The first part

\footnotetext{
* Corresponding author. Tel.: 514-340-4711 Ext. 4007; Fax: 514-340-5867; E-mail: boukas@meca.polymtl.ca

† E-mail: peng.shi@dsto.defence.gov.au

‡E-mail: mehmet@cssip.edu.au

${ }^{\S}$ E-mail: yalcin.kaya@unisa.edu.au
} 
is called as the state of the system which is continuous-valued, and the second part is regarded as the mode of the system which takes discrete values.

The problems of stochastic stability and stochastic stabilizability have been tackled by many authors and recently we have seen the publication of different results ranging from delay-independent to delay-dependent mainly for the continuous-time case such as $[3,10,15]$. Several situations were considered including constant time-delay, time-varyingdelay and mode-dependent time-delay for the continuous-time systems and constant timedelay for discrete-time systems such as [13]. However, to the best of authors' knowledge, the mode-dependent time-delay case for the class of discrete-time linear systems with Markovian jump parameters has not been addressed and this will be the subject of this paper.

The goal of this paper is to develop mode-dependent sufficient conditions for stochastic stability and stochastic stabilizability for linear discrete-time systems with Markovian jump parameters and mode-dependent time-delay in the state. The linear matrix inequality (LMI) techniques described in [4] are used to solve the above problems.

The paper is organized as follows. In Section 2, the problem is stated and the objective of the paper is formulated. The problem of stochastic stability for the given system is examined and delay-dependent sufficient condition is developed in Section 3. We continue in Section 4, to investigate the problem of stabilizability and establish delay-dependent conditions. In addition, a design algorithm that stabilizes the resulting closed-loop system is provided. A numerical example is given in Section 5 to illustrate the proposed theoretical results.

\section{PROBLEM STATEMENT}

Let $\left\{r_{k}, k \geq 0\right\}$ be a homogeneous Markov chain that takes values in a finite state space $\mathcal{S}=\{1,2, \ldots, N\}$ with transition probabilities $p_{i j}$ from mode $i$ to mode $j$ defined by:

$$
p_{i j}=\operatorname{Pr}\left(r_{k+1}=j \mid r_{k}=i\right)
$$

where $p_{i j} \geq 0$ and $\sum_{j=1}^{N} p_{i j}=1$ for all $i, j \in \mathcal{S}$.

The dynamics of the system is assumed to be described by the following set of equations:

$$
\sum:\left\{\begin{array}{l}
x_{k+1}=A\left(r_{k}\right) x_{k}+A_{d}\left(r_{k}\right) x_{k-d_{r_{k}}}+B\left(r_{k}\right) u_{k} \\
x_{l}=\phi_{l}, \quad l=-d_{r_{0}}, \ldots,-1,0 .
\end{array}\right.
$$

the discrete-time index $k$ is assumed to take values $\left[-d_{r_{k}}, \ldots, 0\right]$ and $r_{0}$ is assumed to be given. Here $x_{k}$ and $u_{k}$ are the $n$ - and $m$-dimensional state and control input vectors respectively at instant $k$. The matrices $A\left(r_{k}\right), A_{d}\left(r_{k}\right)$ and $B\left(r_{k}\right)$ are constant matrices of appropriate sizes for any fixed values of $r_{k}$ in $\mathcal{S}$, and $d_{r_{k}}$ is a positive integer representing the time-delay of the system. Note that since $r_{k}$ is a Markov chain, so is $d_{r_{k}}$ with the same transition probabilities. $\phi_{k}$ is some initial condition for the state vector $x_{k}$. We assume in this paper that $d_{r_{k}}$ is mode-dependent.

Our objective in this paper is to obtain sufficient conditions that guarantee the stochastic stability and the stochastic stabilizability of the class of system under consideration.

For the system $\Sigma$ in (2), we use the following control law:

$$
u_{k}=K\left(r_{k}\right) x_{k}
$$

where the control gain $K\left(r_{k}\right)$ is to be designed for each system mode $r_{k} \in \mathcal{S}$.

Notation The notation used in this paper is quite standard. $\mathbb{Z}, \mathbb{R}^{n}$ and $\mathbb{R}^{n \times m}$ denote, respectively, the set of integer numbers, the $n$ dimensional Euclidean space and the set of all $n \times m$ real matrices. The superscript " $\mathrm{T}$ " denotes the transpose and the notation $X \geq Y$ 
(respectively, $X>Y$ ) where $X$ and $Y$ are symmetric matrices, means that $X-Y$ is positive semi-definite (respectively, positive-definite). $P=(P(1), \ldots, P(N))>0$ means all $P(1), \ldots, P(N)$ are symmetric and positive-definite. $I$ is the identity matrix with compatible dimension. $\mathrm{E}\{\cdot\}$ denotes the expectation operator with respective to certain probability measure $P . \ell_{2}[0, \infty]$ is the space of square summable vector sequence over $[0, \infty]$. $\|\cdot\|$ will refer to the Euclidean vector norm whereas $\|\cdot\|_{[0, \infty]}$ denotes the $\ell_{2}[0, \infty]$-norm over $[0, \infty]$ defined as $\|f\|_{[0, \infty]}^{2}=\sum_{0}^{\infty}\left\|f_{k}\right\|^{2}$. For simplicity of presentation, we define $\underline{d}, \bar{d}, \underline{p}$ as:

$$
\begin{aligned}
& \underline{d}=\min \left(d_{1}, \ldots, d_{N}\right) \\
& \bar{d}=\max \left(d_{1}, \ldots, d_{N}\right) \\
& \underline{p}=\min \left(p_{11}, \ldots, p_{N N}\right)
\end{aligned}
$$

For a given set of positive definite matrices $P(j)$ for $j=1, \ldots, N$, we define the following convex combinations $\bar{P}(i)$ as

$$
\bar{P}(i)=\sum_{j=1}^{N} p_{r_{k} j} P(j)
$$

where $i=1, \ldots, N$.

Remark 1 Notice that, due to the existence of time-delay, $d_{r_{k}}$, in the system $\Sigma$, the joint state vector $\left(x_{k}, r_{k}\right)$ is not a Markov chain, however we can define $X_{k}=\left(x_{k-d_{r_{k}}}\right.$, $\left.x_{k-d_{r_{k}}+1}, \ldots, x_{k}\right)$ to overcome this and get a Markov chain, thus it can be seen that $\left(X_{k}, r_{k}\right)$ is a Markov chain.

Remark 2 Indeed, system $\Sigma$ in (2) can be reformulated as a one without time-delay by constructing a higher dimensional vector $X(k)$ defined as:

$$
X(k)=\left[\begin{array}{c}
x(k) \\
x(k-1) \\
x(k-2) \\
\vdots \\
x(k-\underline{d})
\end{array}\right]
$$

Then, system $\Sigma$ can be rewritten as

$$
\begin{aligned}
X(k+1) & =F\left(r_{k}\right) X(k)+G\left(r_{k}\right) u(k) \\
x(k) & =H X(k)
\end{aligned}
$$

where

$$
F\left(r_{k}\right)=\left[\begin{array}{ccccccc}
A\left(r_{k}\right) & 0 & \cdots & A_{d}\left(r_{k}\right) & 0 & \cdots & 0 \\
I & 0 & \cdots & 0 & \cdots & \cdots & 0 \\
0 & I & \cdots & 0 & \cdots & \cdots & 0 \\
\vdots & 0 & \ddots & & & & \vdots \\
\vdots & & & \ddots & & & \vdots \\
\vdots & \vdots & & & \ddots & & \\
0 & 0 & 0 & \cdots & 0 & I & 0
\end{array}\right]
$$




$$
G\left(r_{k}\right)=\left[\begin{array}{c}
B\left(r_{k}\right) \\
0 \\
\vdots \\
0
\end{array}\right], \quad H=\left[\begin{array}{llll}
I & 0 & \cdots & 0
\end{array}\right]
$$

Note that system (6) and (7) is time-delay free, for which, the issues of stochastic stability, stabilization and control can be sorted out by using standard existing results on linear discrete-time Markovian jump systems, for example, Ji et al. [7]. In this paper, instead of using the extending system dimension approach, we propose a different methodology to handle system (2), by which the maximum unknown time-delay can be conventionally determined such that the underlying system remains stochastically stable. Notice that this point is very important since it is difficult to know time-delay exactly in practice, thus using upper and lower bounds in design would be an alternative effective way.

In next section, we consider the stability of the system $\Sigma$ given in (2).

\section{STABILITY}

In this section, we present delay-dependent conditions that can be used to check whether the system $\Sigma$ in (2) is stochastically stable or not. First, we introduce the following stochastic stability concept.

DEFINITION 1 For the system $\Sigma$ in (2) with $u_{k}=0$ for all $k \geq 0$, the equilibrium point 0 is stochastically stable, if for every initial state, $\left(x_{0}, r_{0}\right)$, the following holds:

$$
\mathrm{E}\left\{\sum_{k=0}^{\infty}\left\|x_{k}(\cdot)\right\|^{2} \mid x_{0}, r_{0}\right\}<\infty
$$

In the rest of this paper, we will use $x_{k}$ to denote the solution, $x_{k}\left(x_{0}, r_{0}\right)$ when the initial condition, $\left(x_{0}, r_{0}\right)$ are given. We will also use $X_{k}=\left\{x_{k-d_{r_{k}}}, x_{k-d_{k}+1}, \ldots, x_{k}\right\}$.

The following result concerns with stochastic stability of the system $\Sigma$ in (2).

THEOREM 1 If there exist symmetric and positive-definite matrices $P=(P(1), \ldots, P(N))>0$ and $Q>0$ such that the following holds for every $r_{k} \in \mathcal{S}$ :

$$
M\left(r_{k}\right)=\left[\begin{array}{cc}
M_{11}\left(r_{k}\right) & A^{\top}\left(r_{k}\right) \bar{P}\left(r_{k}\right) A_{d}\left(r_{k}\right) \\
A_{d}^{\top}\left(r_{k}\right) \bar{P}\left(r_{k}\right) A\left(r_{k}\right) & A_{d}^{\top}\left(r_{k}\right) \bar{P}\left(r_{k}\right) A_{d}\left(r_{k}\right)-Q
\end{array}\right]<0
$$

with $M_{11}\left(r_{k}\right)=A^{\top}\left(r_{k}\right) \bar{P}\left(r_{k}\right) A\left(r_{k}\right)-P\left(r_{k}\right)+(1+(\bar{d}-\underline{d})(1-\underline{p})) Q$, then the system $\Sigma$ in $(2)$ is stochastically stable.

Proof To prove our theorem, let us consider the following Lyapunov candidate functional:

$$
V\left(X_{k}, k\right)=V_{1}\left(X_{k}, k\right)+V_{2}\left(X_{k}, k\right)+V_{3}\left(X_{k}, k\right)
$$


with

$$
\begin{aligned}
& V_{1}\left(X_{k}, k\right)=x_{k}^{\top} P\left(r_{k}\right) x_{k} \\
& V_{2}\left(X_{k}, k\right)=\sum_{l=k-d_{r_{k}}}^{k-1} x_{l}^{\top} Q x_{l} \\
& V_{3}\left(X_{k}, k\right)=\sum_{l=-\bar{d}}^{-\underline{d}} \sum_{m=k+l+1}^{k-1} x_{m}^{\top} \bar{Q} x_{m},
\end{aligned}
$$

where $\bar{Q}=(1-\underline{p}) Q$.

$\Delta V\left(X_{k}, k\right)$ is given by:

$$
\Delta V\left(X_{k}, k\right)=\Delta V_{1}\left(X_{k}, k\right)+\Delta V_{2}\left(X_{k}, k\right)+\Delta V_{3}\left(X_{k}, k\right)
$$

Let us now compute $\Delta V_{1}\left(X_{k}, k\right)$ :

$$
\begin{aligned}
\Delta V_{1}\left(X_{k}, k\right)= & \mathrm{E}\left[V_{1}\left(X_{k+1}, k+1\right) \mid X_{k}, r_{k}\right]-V_{1}\left(X_{k}, k\right) \\
= & \mathrm{E}\left[x_{k+1}^{\top} P\left(r_{k+1}\right) x_{k+1} \mid X_{k}, r_{k}\right]-x_{k}^{\top} P\left(r_{k}\right) x_{k} \\
= & x_{k}^{\top}\left[A^{\top}\left(r_{k}\right) \bar{P}\left(r_{k}\right) A\left(r_{k}\right)-P\left(r_{k}\right)\right] x_{k}+x_{k}^{\top} A^{\top}\left(r_{k}\right) \bar{P}\left(r_{k}\right) A_{d}\left(r_{k}\right) x_{k-d_{r_{k}}} \\
& +x_{k-d_{r_{k}}} A_{d}^{\top} \bar{P}\left(r_{k}\right) A\left(r_{k}\right) x_{k}+x_{k-d_{r_{k}}} A_{d}^{\top}\left(r_{k}\right) \bar{P}\left(r_{k}\right) A_{d}\left(r_{k}\right) x_{k-d_{r_{k}}} .
\end{aligned}
$$

For $V_{2}\left(X_{k}, k\right)$, by standard manipulation, we have:

$$
\begin{aligned}
\Delta V_{2}\left(X_{k}, k\right) & =\mathrm{E}\left[V_{2}\left(X_{k+1}, k+1\right) \mid X_{k}, r_{k}\right]-V_{2}\left(X_{k}, k\right) \\
& =\mathrm{E}\left[\sum_{l=k+1-d_{r_{k+1}}}^{k} x_{l}^{\top} Q x_{l} \mid X_{k}, r_{k}\right]-\sum_{l=k-d_{r_{k}}}^{k-1} x_{l}^{\top} Q x_{l} .
\end{aligned}
$$

By using the fact that the Markov chain at time $(k+1)$ can either remain at mode $r_{k}$ or jumps to mode $j$, we get the following:

$$
\begin{aligned}
\Delta V_{2}\left(X_{k}, k\right)= & p_{r_{k} r_{k}} \sum_{l=k+1-d_{r_{k}}}^{k} x_{l}^{\top} Q x_{l}-p_{r_{k} r_{k}} \sum_{l=k-d_{r_{k}}}^{k-1} x_{l}^{\top} Q x_{l} \\
& +\sum_{j \neq r_{k}}^{N} p_{r_{k} j} \sum_{l=k+1-d_{j}}^{k} x_{l}^{\top} Q x_{l}-\sum_{j \neq r_{k}}^{N} p_{r_{k} j} \sum_{l=k-d_{r_{k}}}^{k-1} x_{l}^{\top} Q x_{l}
\end{aligned}
$$

After simple algebraic manipulation we can rewrite $\Delta V_{2}\left(X_{k}, k\right)$ as follows:

$$
\begin{aligned}
\Delta V_{2}\left(X_{k}, k\right)= & p_{r_{k} r_{k}}\left[x_{k}^{\top} Q x_{k}-x_{k-d_{r_{k}}}^{\top}-Q x_{k-d_{r_{k}}}^{\top}\right] \\
& +\sum_{j \neq r_{k}}^{N} p_{r_{k} j}\left[x_{k}^{\top} Q x_{k}-x_{k-d_{r_{k}}}^{\top} Q x_{k-d_{r_{k}}}-\sum_{l=k+1-d_{j}}^{k-d_{r_{k}}} x_{l}^{\top} Q x_{l}\right] \\
= & x_{k}^{\top} Q x_{k}-x_{k-d_{r_{k}}}^{\top} Q x_{k-d_{r_{k}}}+\sum_{j \neq r_{k}}^{N} p_{r_{k} j} \sum_{l=k+1-d_{j}}^{k-d_{r_{k}}} x_{l}^{\top} Q x_{l} .
\end{aligned}
$$


Bearing in mind that $\sum_{j \neq r_{k}}^{N} p_{r_{k} j}=1-p_{r_{k} r_{k}}$ and the bounds on the time-delay and on $p_{r_{k} r_{k}}$, we have the following expression for $\Delta V_{2}\left(X_{k}, k\right)$ :

$$
\Delta V_{2}\left(X_{k}, k\right) \leq x_{k}^{\top} Q x_{k}-x_{k-d_{r_{k}}}^{\top} Q x_{k-d_{r_{k}}}+(1-\underline{p}) \sum_{l=k+1-\bar{d}}^{k-\underline{d}+1} x_{l}^{\top} Q x_{l} .
$$

In terms of $V_{3}\left(X_{k}, k\right)$, one has:

$$
\begin{aligned}
\Delta V_{3}\left(X_{k}, k\right) & =\mathrm{E}\left[V_{3}\left(X_{k+1}, k+1\right) \mid X_{k}, k\right]-V_{3}\left(X_{k}, k\right) \\
& =\mathrm{E}\left[\sum_{l=-\bar{d}}^{\underline{-d}} \sum_{m=k+2+l}^{k} x_{m}^{\top} \bar{Q} x_{m} \mid X_{k}, r_{k}\right]-\sum_{l=-\bar{d}}^{-\underline{d}} \sum_{m=k+l+1}^{k-1} x_{m}^{\top} \bar{Q} x_{m} \\
& =\sum_{l=-\bar{d}}^{-\underline{d}}\left[x_{k}^{\top} \bar{Q} x_{k}-x_{k+l+1}^{\top} \bar{Q} x_{k+l+1}\right]=(\bar{d}-\underline{d}) x_{k}^{\top} \bar{Q} x_{k}-\sum_{l=-\bar{d}}^{-\underline{d}} x_{k+1+l}^{\top} Q x_{k+1+l} \\
& =(\bar{d}-\underline{d}) x_{k}^{\top} \bar{Q} x_{k}-\sum_{l=k-\bar{d}+1}^{k-\underline{d}+1} x_{l}^{\top} \bar{Q} x_{l} .
\end{aligned}
$$

Finally, by using equalities (12) and (13), inequality (14), and the fact that $\bar{Q}=(1-\underline{p}) Q$, together with the condition (11), we obtain

$$
\Delta V\left(X_{k}, k\right) \leq\left[\begin{array}{ll}
x_{k}^{\top} & x_{k-d_{r_{k}}}^{\top}
\end{array}\right] M\left(r_{k}\right)\left[\begin{array}{c}
x_{k} \\
x_{k-d_{r_{k}}}
\end{array}\right]<0 .
$$

The rest of the proof follows the same lines as the ones given in Boukas and Yang [1] or Boukas and Shi [2].

Remark 3 It is of practical interest to find the maximum time-delay such that the system is stable. The maximum time-delay can be obtained by solving the following linear optimization problem:

$$
\max _{\substack{\bar{d}>0, P=(P(1), \ldots, P(N))>0, Q>0 \\ M\left(r_{k}\right)<0}} \bar{d}
$$

where the last constraint $M\left(r_{k}\right)<0$ was given in (11).

When time-delay is constant and mode independent, i.e. $d_{r_{k}}=d$ for all $r_{k} \in \mathcal{S}$ and therefore $d=\bar{d}=\underline{d}$, the results given by Theorem 1 is replaced by the following result.

COROLlary 1 If there exist symmetric and positive-definite matrices $P=(P(1), \ldots$, $P(N))>0$ and $Q>0$ such that the following holds for every $r_{k} \in \mathcal{S}$ :

$$
N\left(r_{k}\right)=\left[\begin{array}{cc}
N_{11}\left(r_{k}\right) & A^{\top}\left(r_{k}\right) \bar{P}\left(r_{k}\right) A_{d}\left(r_{k}\right) \\
A_{d}^{\top}\left(r_{k}\right) \bar{P}\left(r_{k}\right) A\left(r_{k}\right) & A_{d}^{\top}\left(r_{k}\right) \bar{P}\left(r_{k}\right) A_{d}\left(r_{k}\right)-Q
\end{array}\right]<0
$$

where $N_{11}\left(r_{k}\right)=A^{\top}\left(r_{k}\right) \bar{P}\left(r_{k}\right) A\left(r_{k}\right)-P\left(r_{k}\right)+Q$, then the system $\Sigma$ in (2) is stochastically stable. 


\section{STABILIZABILITY}

Our goal in this section is to design a state-feedback controller which stabilizes the resulting closed-loop of system. The controller is supposed to have the form of (3). Let us first define the concept of stochastic stabilizability.

DEFINITION 2 System (2) is stochastically stabilizable, if for every initial state there exists a state-feedback controller (3) with gain $K=(K(1), \ldots, K(N))$ such that the resulting closedloop system is stochastically stable.

Replacing the control $u_{k}$ by its expression given by Eq. (3) and substituting it into system $\Sigma$ in (2), we get the following dynamics for the closed-loop system $\tilde{\Sigma}$ :

$$
\tilde{\Sigma}:\left\{\begin{array}{l}
x_{k+1}=\left[A\left(r_{k}\right)+B\left(r_{k}\right) K\left(r_{k}\right)\right] x_{k}+A_{d}\left(r_{k}\right) x_{k-d_{r_{k}}} \\
x_{l}=\phi_{l}, \quad l=-d_{r_{0}}, \ldots,-1,0
\end{array}\right.
$$

If we let $\bar{A}\left(r_{k}\right)=A\left(r_{k}\right)+B\left(r_{k}\right) K\left(r_{k}\right)$, we have the following result using the previous result on stochastic stability.

THEOREM 2 For a given set of gains $K=(K(1), \ldots, K(N))$, if there exist symmetric and positive-definite matrices $P=(P(1), \ldots, P(N))>0$ and $Q>0$ such that the following holds for every $r_{k} \in \mathcal{S}$ :

$$
\mathcal{M}\left(r_{k}\right)=\left[\begin{array}{cc}
\mathcal{M}_{11}\left(r_{k}\right) & \bar{A}^{\top}\left(r_{k}\right) \bar{P}\left(r_{k}\right) A_{d}\left(r_{k}\right) \\
A_{d}^{\top}\left(r_{k}\right) \bar{P}\left(r_{k}\right) \bar{A}\left(r_{k}\right) & A_{d}^{\top}\left(r_{k}\right) \bar{P}\left(r_{k}\right) A_{d}\left(r_{k}\right)-Q
\end{array}\right]<0
$$

where $\mathcal{M}_{11}\left(r_{k}\right)=\bar{A}^{\top}\left(r_{k}\right) \bar{P}\left(r_{k}\right) \bar{A}\left(r_{k}\right)-P\left(r_{k}\right)+(1+(\bar{d}-\underline{d})(1-\underline{p})) Q$, then the system $\tilde{\Sigma}$ in (17) is stochastically stable under the state feedback control law (3).

Proof It can be worked out along the same line as in Theorem 1.

Similarly, when the time-delay is constant and does not depend on the mode, i.e. $d_{r_{k}}=d$ for all $r_{k} \in \mathcal{S}$ and therefore $d=\bar{d}=\underline{d}$. Theorem 2 is reduced to the following corollary.

COROLLARY 2 For a given set of gains $K=(K(1), \ldots, K(N))$, if there exist symmetric and positive-definite matrices $P=(P(1), \ldots, P(N))>0$ and $Q>0$ such that the following holds for every $r_{k} \in \mathcal{S}$ :

$$
\mathcal{N}\left(r_{k}\right)=\left[\begin{array}{cc}
\mathcal{N}_{11}\left(r_{k}\right) & \bar{A}^{\top}\left(r_{k}\right) \bar{P}\left(r_{k}\right) A_{d}\left(r_{k}\right) \\
A_{d}^{\top}\left(r_{k}\right) \bar{P}\left(r_{k}\right) \bar{A}\left(r_{k}\right) & A_{d}^{\top}\left(r_{k}\right) \bar{P}\left(r_{k}\right) A_{d}\left(r_{k}\right)-Q
\end{array}\right]<0,
$$

where $\mathcal{N}_{11}\left(r_{k}\right)=\bar{A}^{\top}\left(r_{k}\right) \bar{P}\left(r_{k}\right) \bar{A}\left(r_{k}\right)-P\left(r_{k}\right)+Q$, then the system $\Sigma$ in (2) is stochastically stable under the control (3). 
Now, we are in the position to design the controller, $u_{k}=K x_{k}$ in Theorem (2), that stabilizes systems (2). First, notice that, if we define:

$$
\begin{aligned}
W\left(r_{k}\right) & =\left(\sqrt{p_{r_{k}}} I, \ldots, \sqrt{\left.p_{r_{k} N} I\right)}\right. \\
\mathcal{Z} & =\operatorname{diag}(Z(1), \ldots, Z(N)) \\
\rho & =1+(1-\underline{p})(\bar{d}-\underline{d})
\end{aligned}
$$

with $Z\left(r_{k}\right)=P^{-1}\left(r_{k}\right)$, the right hand side of (18) can be rewritten as:

$$
\begin{aligned}
& {\left[\begin{array}{cc}
-P\left(r_{k}\right)+\rho Q & 0 \\
0 & -Q
\end{array}\right]+\left[\begin{array}{c}
\bar{A}^{\top}\left(r_{k}\right) W\left(r_{k}\right) \\
A_{d}^{\top}\left(r_{k}\right) W\left(r_{k}\right)
\end{array}\right]\left[\begin{array}{ccc}
P(1) & & \\
& \ddots & \\
& & P(N)
\end{array}\right]} \\
& \times\left[\begin{array}{ll}
W^{\top}\left(r_{k}\right) \bar{A}\left(r_{k}\right) & \left.W^{\top}\left(r_{k}\right) A_{d}\left(r_{k}\right)\right]
\end{array}\right.
\end{aligned}
$$

by using Schur complement, the following inequality can be obtained from (20):

$$
\left[\begin{array}{ccc}
-P\left(r_{k}\right)+\rho Q & 0 & \bar{A}^{\top}\left(r_{k}\right) W\left(r_{k}\right) \\
0 & -Q & A_{d}^{\top}\left(r_{k}\right) W\left(r_{k}\right) \\
W^{\top}\left(r_{k}\right) \bar{A}\left(r_{k}\right) & W^{\top}\left(r_{k}\right) A_{d}\left(r_{k}\right) & -\mathcal{Z}
\end{array}\right]<0
$$

Let $U=Q^{-1}$ and $Y\left(r_{k}\right)=K\left(r_{k}\right) Z\left(r_{k}\right)$. Pre- and post-multiplying inequality (21) by $\operatorname{diag}\left(Z\left(r_{k}\right), U, I\right)$ we get:

$$
\left[\begin{array}{ccc}
-Z\left(r_{k}\right)+Z\left(r_{k}\right) \rho Q Z\left(r_{k}\right) & 0 & Z\left(r_{k}\right) \bar{A}^{\top}\left(r_{k}\right) W\left(r_{k}\right) \\
0 & -U & U A_{d}^{\top}\left(r_{k}\right) W\left(r_{k}\right) \\
W^{\top}\left(r_{k}\right) \bar{A}\left(r_{k}\right) Z\left(r_{k}\right) & W^{\top}\left(r_{k}\right) A_{d}\left(r_{k}\right) U & -\mathcal{Z}
\end{array}\right]<0 .
$$

Using now the fact that $Y\left(r_{k}\right)=K\left(r_{k}\right) Z\left(r_{k}\right)$, together with Schur complement again, one has:

$$
\left[\begin{array}{cccc}
-Z\left(r_{k}\right) & 0 & \left(A\left(r_{k}\right) Z\left(r_{k}\right)\right. & Z\left(r_{k}\right) \\
0 & -U & U A_{d}^{\top}\left(r_{k}\right) W\left(r_{k}\right) & 0 \\
W^{\top}\left(r_{k}\right)\left(A\left(r_{k}\right) Z\left(r_{k}\right)\right. & W^{\top}\left(r_{k}\right) A_{d}\left(r_{k}\right) U & -\mathcal{Z} & 0 \\
\left.+B\left(r_{k}\right) Y\left(r_{k}\right)\right) & 0 & 0 & -\frac{1}{\rho} U
\end{array}\right]<0
$$

To summarize the above analysis, a control design algorithm that stabilizes the system $\Sigma$ in (2) is given by the following theorem. 
THEOREM 3 If there exist symmetric and positive-definite matrices $\mathcal{Z}=(Z(1), \ldots$, $Z(N))>0, \mathcal{Y}=(Y(1), \ldots, Y(N))>0$ and $U>0$ such that the following holds for each $r_{k} \in \mathcal{S}:$

$$
\left[\begin{array}{cccc}
-Z\left(r_{k}\right) & 0 & \left(A\left(r_{k}\right) Z\left(r_{k}\right)\right. & Z\left(r_{k}\right) \\
0 & -U & \left.+B\left(r_{k}\right) Y\left(r_{k}\right)\right)^{\top} W(r k) & \\
W^{\top}\left(r_{k}\right)\left(A\left(r_{k}\right) Z\left(r_{k}\right)\right. & W^{\top}\left(r_{k}\right) A_{d}\left(r_{k}\right) U & -\mathcal{Z}\left(r_{k}\right) W\left(r_{k}\right) & 0 \\
\left.+B\left(r_{k}\right) Y\left(r_{k}\right)\right) & 0 & 0 & 0 \\
Z\left(r_{k}\right) & & & -\frac{1}{\rho} \mathrm{U}
\end{array}\right]<0
$$

then, the system $\Sigma$ in (2) is stochastically stable under the controller (3) and the control gain is given by $K\left(r_{k}\right)=Y\left(r_{k}\right) Z^{-1}\left(r_{k}\right)$.

Similar to Corollary 2, if the time-delay $d_{r_{k}}$ is constant and independent of system mode, Theorem 3 becomes:

COROLLARY 3 If there exist symmetric and positive-definite matrices $\mathcal{Z}=(Z(1), \ldots$, $Z(N))>0, \mathcal{Y}=(Y(1), \ldots, Y(N))>0$ and $U>0$ such that the following holds for each $r_{k} \in \mathcal{S}:$

$$
\left[\begin{array}{cccc} 
& \left(A\left(r_{k}\right) Z\left(r_{k}\right)\right. & \\
-Z\left(r_{k}\right) & 0 & \left.+B\left(r_{k}\right) Y\left(r_{k}\right)\right)^{\top} & Z\left(r_{k}\right) \\
& & \times W(r k) & \\
W^{\top}\left(r_{k}\right)\left(A\left(r_{k}\right) Z\left(r_{k}\right)\right. & W^{\top}\left(r_{k}\right) A_{d}\left(r_{k}\right) U & -\mathcal{Z} & 0 \\
\left.+B\left(r_{k}\right) Y\left(r_{k}\right)\right) & 0 & 0 & -U
\end{array}\right]<0
$$

then, the system $\Sigma$ in (2) is stochastically stable under the controller (3) and the control gain is given by $K\left(r_{k}\right)=Y\left(r_{k}\right) Z^{-1}\left(r_{k}\right)$.

Remark 4 To find the maximal bound of time delay $\bar{d}$ with which system (2) is stochastically stable, we need to solve the following optimization problem:

$$
\max _{\substack{\bar{d}>0, \mathcal{Z}=(Z(1), \ldots, Z(N))>0, \mathcal{Y}=(Y(1) \ldots, Y(N))>0, U>0}} \quad\{\bar{d}\}
$$

such that the constraint (24) is satisfied. 


\section{NUMERICAL EXAMPLE}

In this section, we consider the stabilizability problem of a 2-D Markovian jump linear systems with mode dependant time delays to illustrate the results presented earlier.

Let us consider a system described by (2) and suppose that the system data are as follows: The system has two modes, i.e., $\mathcal{S}=\{1,2\}$. The mode switching is governed by a Markov chain that has the following transition probability matrix:

$$
\Lambda=\left(\begin{array}{cc}
0.5 & 0.5 \\
0.3 & 0.7
\end{array}\right) \text {. }
$$

Let us now, see how we can use the design algorithm of Theorem 3 , to determine the controller gain $K=(K(1), K(2))$. For this purpose let us consider the following data:

$$
\begin{aligned}
A(1) & =\left[\begin{array}{cc}
-1.8 & 0.11 \\
0 & 0.26
\end{array}\right] \\
A(2) & =\left[\begin{array}{cc}
0.3 & 0 \\
0.16 & 0.4
\end{array}\right] \\
A_{d}(1) & =\left[\begin{array}{cc}
0.19 & 0 \\
0 & -0.1
\end{array}\right] \\
A_{d}(2) & =\left[\begin{array}{cc}
0.2 & 0 \\
0.3 & 0.2
\end{array}\right] \\
B(1) & =\left[\begin{array}{l}
1 \\
0
\end{array}\right] \\
B(2) & =\left[\begin{array}{l}
0 \\
1
\end{array}\right] \\
\bar{\tau} & =2, \quad \underline{\tau}=1 .
\end{aligned}
$$

Using this data, solving LMIs (24) gives the following feasible solution:

$$
\begin{aligned}
Z(1) & =\left[\begin{array}{cc}
26.3572 & 2.8503 \\
2.8503 & 61.6519
\end{array}\right]>0 \\
Z(2) & =\left[\begin{array}{ll}
70.5968 & -1.8900 \\
-1.8900 & 64.0583
\end{array}\right]>0 \\
Y(1) & =\left[\begin{array}{ll}
34.4743 & -0.6956
\end{array}\right] \\
Y(2) & =\left[\begin{array}{ll}
-8.2613 & -17.0265
\end{array}\right] \\
U & =\left[\begin{array}{ll}
119.3331 & -0.3912 \\
-0.3912 & 121.0920
\end{array}\right]>0
\end{aligned}
$$

which gives the following gains:

$$
\begin{aligned}
& K(1)=\left[\begin{array}{ll}
1.3158 & -0.0721
\end{array}\right] \\
& K(2)=\left[\begin{array}{ll}
-0.1242 & -0.2695
\end{array}\right]
\end{aligned}
$$


Therefore, controller (3) with gains

$$
K(1)=\left[\begin{array}{ll}
1.3158 & -0.0721
\end{array}\right], \quad K(2)=\left[\begin{array}{ll}
-0.1242 & -0.2695
\end{array}\right]
$$

stabilizes the system under study in the MSQS sense.

\section{CONCLUSION}

In this paper, we have studied the problems of stochastic stability and stochastic stabilizability for a class of linear discrete time systems with Markovian jump parameters and mode-dependent time-delay. Sufficient conditions in the LMI formalism have been developed to solve the above problems. An algorithm to design the stabilizing controller is also provided. A numerical example is included to demonstrate the potential of the proposed techniques.

\section{References}

[1] Boukas, E. K. and Yang, H. (1995) Stability of discrete-time linear systems with Markovian jumping parameters, Math. of Control, Signals and Systems, 8, 390-402.

[2] Boukas, E. K. and Shi, P. (1997) $H_{\infty}$ control for discrete-time linear systems with Markovian jumping parameters, In: Proc. 36th IEEE Conf. on Decision and Control. San Diego, Cali, pp. 4143-4148

[3] Boukas, K. and Liu, Z. (2002) Deterministic and Stochastic Time-delay Systems. Birkhauser, Boston.

[4] Boyd, S., Ghaoui, L. E., Feron, E. and Balakrishnan, V. (1994) Linear Matrix Inequalities in System and Control Theory. SIAM: Philadelphia.

[5] Gutman, S. and Palmor, Z. (1982) Properties of min-max controllers in uncertain dynamical systems, SIAM J. Contr. and Optimiz., 20, 850-861.

[6] Hasanul Basher, A. M. and Mukundanand, R. and O'Connor, D. A. (1986) Memoryless feedback control in uncertaindynamic delay systems, Int. J. Syst. Sci., 17, 409-415.

[7] Ji, Y., Chizeck, H. J., Feng, X. and Loparo, K. A. (1991) Stability and control of discrete-time jump linear systems, Control Theory and Advanced Technology, 7(2), 247-270.

[8] Li, X. and de Souza, C. E. (1997) Delay-dependent robust stability and stabilization of uncertain linear delay systems: a linear matrix inequality approach, IEEE Trans. Automat. Control, 42(8), 1144-1148.

[9] Mahmoud, M. S. and Al-Muthairi, N. F. (1994) Quadratic stabilization of continuous time systems with statedelay and norm-bounded time-varying uncertainties, IEEE Trans. Automat. Control, 39(10), 2135-2139.

[10] Mahmoud, M. S. (1999) Robust Control and Filtering for Time-delay Systems. Marcel-Dekker: New York.

[11] Malek-Zavarei, M. and Jamshidi, M. (1987) Time-delay systems: analysis, optimization and applications, North-Holland Systems and Control Series.

[12] Nguang, S. K. (1994) Robust stabilization for a class of time-delay nonlinear systems, IEE Proceedings-D, 141(5), 285-288.

[13] Shi, P., Boukas, E. K. and Agarwal, R. K. (1999) Control of Markovian jump discrete-time systems with norm bounded uncertainty and unknown delays, IEEE Trans. Automat. Control, 44(11), 2139-2144.

[14] Song, S. H. and Kim, J. K. (1998) $H_{\infty}$ control of discrete-time linear systems with norm-bounded uncertainties and time-delay in state, Automatica, 34(1), 137-139.

[15] de Souza, C. and Li, X. (1999) Delay dependent robust $H_{\infty}$ control of uncertain linear state-delayed systems, Automatica, 35(8), 1313-1321. 


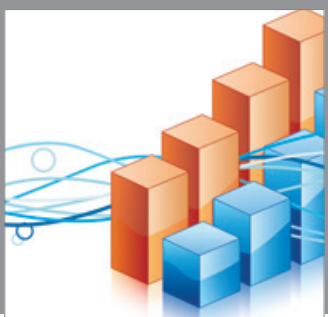

Advances in

Operations Research

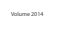

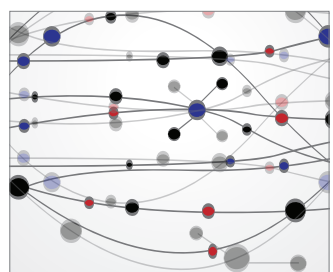

\section{The Scientific} World Journal
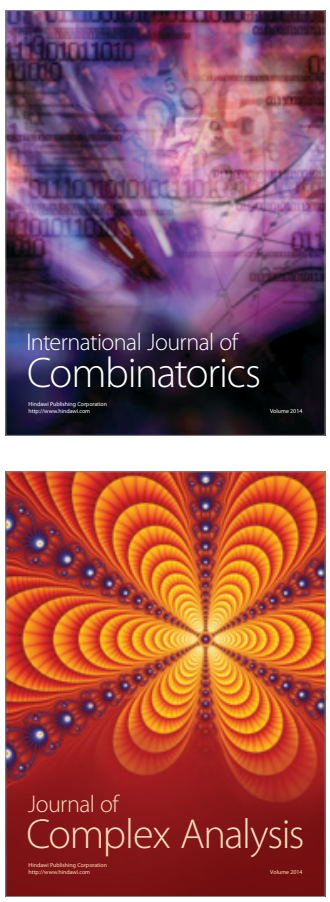

International Journal of

Mathematics and

Mathematical

Sciences
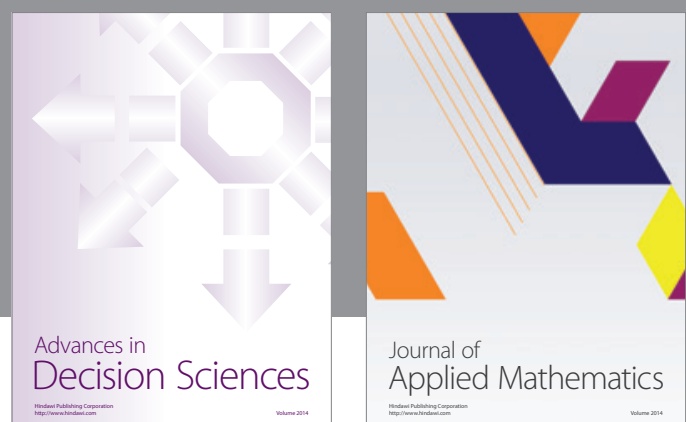

Journal of

Applied Mathematics
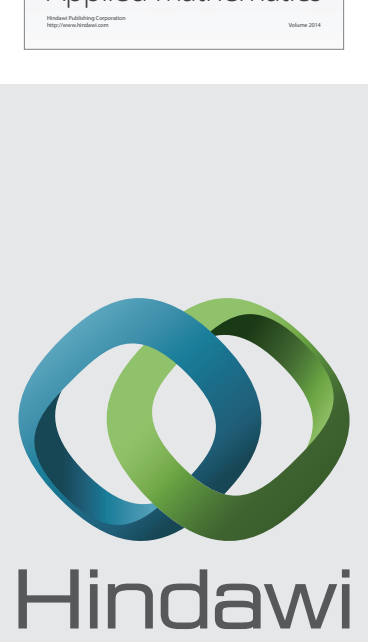

Submit your manuscripts at http://www.hindawi.com
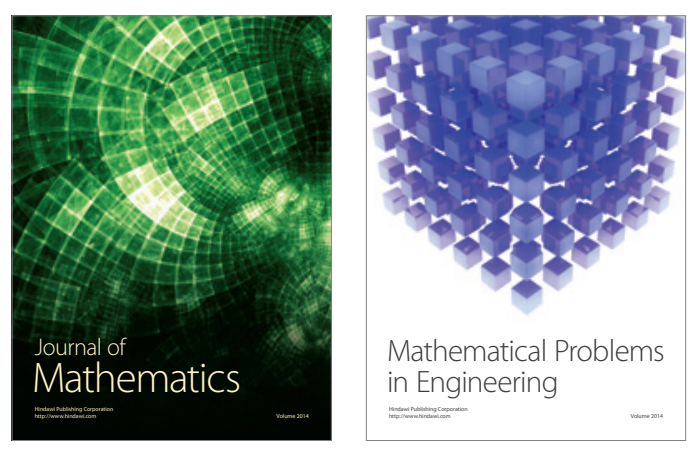

Mathematical Problems in Engineering
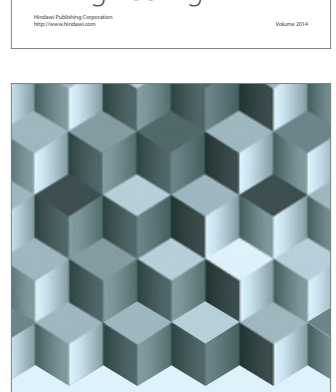

Journal of

Function Spaces
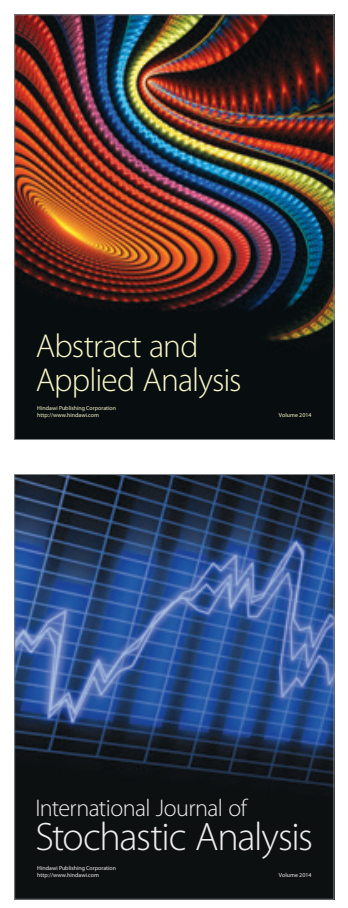

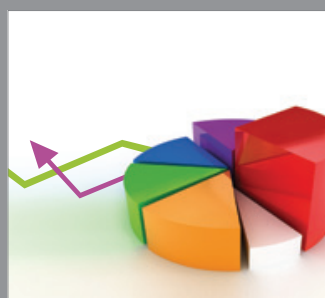

ournal of

Probability and Statistics

Promensencen
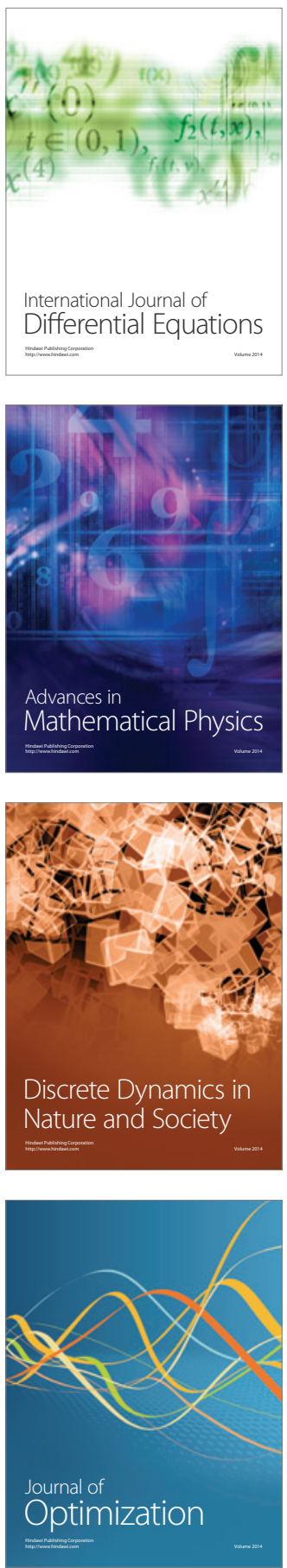